\title{
A Simple Approximation for Axially Symmetric Diffraction of Plane Shocks by Cones
}

Shanbing $\mathrm{Yu}^{*}$ and Hans Grönig

Stoßwellenlabor, Rheinisch-Westfälische Technische Hochschule Aachen

Z. Naturforsch. 39 a, 320-324 (1984); received January 28, 1984

By Whitham's shock dynamics, axially symmetric diffraction problems of plane shocks by cones are reduced to the integration of a system of ordinary differential equations. This paper gives a simpler approach. Approximating the Mach stems by circular arcs, the problems are reduced to the solution of algebraical equations, and the calculating is much simplified. In the cases of small or large cone angles the estimation is even easier.

\section{The Differential Equations Based on Whitham's Shock Dynamics}

Whitham [1,2] has presented an approximate theory - shock dynamics - which describes the propagation of shocks. In axially symmetric diffraction problems of plane shocks by cones, the only parameters prescribed in the problem are the initial Mach number $M_{0}$ and the cone angle $\theta_{\mathrm{w}}$; there is no characteristic length. Hence the solution is selfsimilar, the shock Mach number $M$ and the ray angle $\theta$ must be functions of the single variable $\eta$, the angle coordinate (see Figure 1). Whitham has

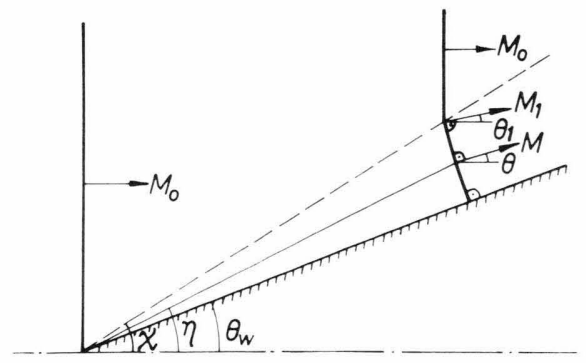

Fig. 1. Diffraction of plane shocks by cones. given the following differential equations for the present problem based on his shock dynamics ([2], (52), (53)).

$$
\begin{aligned}
\frac{1}{M} \frac{\mathrm{d} M}{\mathrm{~d} \eta} & =\tan (\eta-\theta) \frac{\mathrm{d} \theta}{\mathrm{d} \eta}, \\
-\frac{1}{A} \frac{\mathrm{d} A}{\mathrm{~d} \eta} & =\left(-\frac{M}{A} \frac{\mathrm{d} A}{\mathrm{~d} M}\right) \frac{1}{M} \frac{\mathrm{d} M}{\mathrm{~d} \eta} \\
= & \left\{\frac{\mathrm{d} \theta}{\mathrm{d} \eta}+\frac{\tan \theta}{\sin \eta \cos \eta(1+\tan \eta \tan \theta)}\right\} \\
& \cdot \cot (\eta-\theta),
\end{aligned}
$$

where $A$ is the ray area, which represents a known function of $M$

$$
A=f(M)
$$

(its particular form may be seen in [3], p. 8).

At the shock-shock, where $\eta=\chi$ (with $\chi$ the shock-shock angle), the following shock-shock relations must be satisfied ([2], (54))

$$
\theta=\theta_{1}, \quad M=M_{1},
$$

$$
\left.\begin{array}{rl}
\tan \theta_{1}= & {\left[\left(\frac{M_{1}}{M_{0}}\right)^{2}-1\right]^{1 / 2}\left[1-\left(\frac{A_{1}}{A_{0}}\right)^{2}\right]^{1 / 2} /\left(\frac{A_{1}}{A_{0}} \frac{M_{1}}{M_{0}}+1\right)} \\
& \tan \chi=\left[\left(\frac{M_{1}}{M_{0}}\right)^{2}-1\right]^{1 / 2} /\left[1-\left(\frac{A_{1}}{A_{0}}\right)^{2}\right]^{1 / 2}
\end{array}\right\} \text { at } \eta=\chi,
$$

\footnotetext{
* Institute of Mechanics, Academia Sinica, Beijing, People's Republic of China.

Reprint requests to Prof. Dr. H. Grönig, Stoßwellenlabor, Rheinisch-Westfälische Technische Hochschule Aachen, Templergraben 55, D-5100 Aachen.
}

where subscript 1 represents the values at the shock-

shock. At the wall of the cone

$$
\theta=\theta_{\mathrm{w}} \text { for } \eta=\theta_{\mathrm{w}} .
$$

0340-4811/84/0400-0320\$01.3 0/0. - Please order a reprint rather than making your own copy. 
The procedure for finding solutions for a given $M_{0}$ is to choose a value for $M_{1}$, then $\chi$ and $\theta_{1}$ can be found from the shock-shock relations (4). From this point $\left(\eta=\chi, \theta=\theta_{1}, M=M_{1}\right)$ the equations (1), (2) are integrated over the curved shock unto $\theta=\eta$. This common value represents the cone angle $\theta_{\mathrm{w}}$, which corresponds to the chosen value of $M_{1}$. Hence the solution of the problem is reduced to the numerical integration of a system of ordinary differential equations.

\section{Bryson's and Gross' Approximation for Large Cone Angles}

To simplify the solution of (1)-(3), Bryson and Gross [3] have applied straight lines to approximate to the shock shape in a meridian plane, namely it is regarded as if there is a uniform state in the disturbed region. If the shock-shock relations are applied, the obtained relations are the solutions for the diffraction of plane shocks by wedges (Figure 2).

$$
\left.\begin{array}{l}
\frac{M_{1}}{M_{0}}=\frac{\cos \left(\chi-\theta_{1}\right)}{\cos \chi}, \\
\frac{A_{1}}{A_{0}}=\frac{\sin \left(\chi-\theta_{1}\right)}{\sin \chi} .
\end{array}\right\}
$$

Considering the effect of area variation in the case of axial symmetry, the second equation is changed to ([3], (2))

$$
\left.\begin{array}{l}
\frac{M}{M_{0}}=\frac{\cos \left(\chi-\theta_{\mathrm{w}}\right)}{\cos \chi}, \\
\frac{A}{A_{0}}=\frac{\sin \left(\chi-\theta_{\mathrm{w}}\right)}{\sin \chi}\left[1+\frac{\sin \theta_{\mathrm{w}} \cos \left(\chi-\theta_{\mathrm{w}}\right)}{\sin \chi}\right] .
\end{array}\right\}
$$

For $M \gg 1$, (3) yields $A / A_{0}=\left(M_{0} / M\right)^{n}$ with $n=$ 5.0743 , the calculated result of this approximation

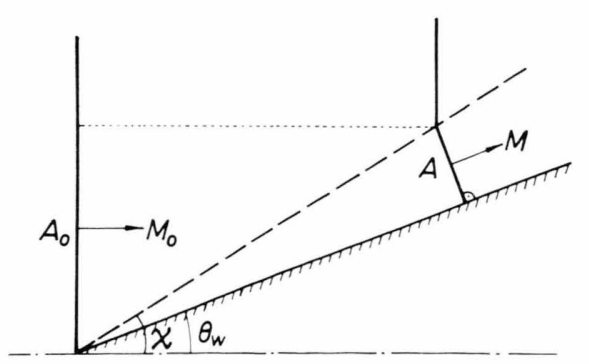

Fig. 2. Straight Mach shock shape approximation. has been plotted in Fig. 3 (see also [3], Figure 11). Comparing this curve (6) with the result of the numerical integration of (1) and (2), the agreement is good only for large cone angles, because the Mach shock in the disturbed region is straight in that case.

\section{Whitham's Small Perturbation Solution}

Whitham has given a small perturbation solution for little cone angles ([2], (74), (75), (76)):

$$
\left.\begin{array}{l}
\chi=m_{0}+\frac{3}{2} B\left(B^{2}+1\right)\left\{1+\frac{M_{0}}{B}\left(\frac{\mathrm{d} m}{\mathrm{~d} M}\right)\right\}_{0}^{2} \theta_{\mathrm{w}}^{4}, \\
\theta_{1}=3 B\left(B^{2}+1\right)\left\{1+\frac{M_{0}}{B}\left(\frac{\mathrm{d} m}{\mathrm{~d} M}\right)_{0}\right\} \theta_{\mathrm{w}}^{4}, \\
\frac{M_{1}-M_{0}}{M_{0}}=3\left(B^{2}+1\right)\left\{1+\frac{M_{0}}{B}\left(\frac{\mathrm{d} m}{\mathrm{~d} M}\right)_{0}\right\} \theta_{\mathrm{w}}^{4},
\end{array}\right\}
$$

where $B=\left[-\frac{M}{A} \frac{\mathrm{d} A}{\mathrm{~d} M}\right]_{M=M_{0}}^{1 / 2}$ is a constant depending on $M_{0}([2],(19))$ and $m$ is a known function of $M$ ([2], (46)). The result for $M \gg 1$ has been plotted also in Figure 3.

\section{Arc Approximation of the Mach Shock Shape}

Here we give an approximation for all cases. In the problem of diffraction of plane shocks by cones, the shock shape in a meridian plane cannot be any straight line except for the plane shock, because there is the effect from the axial symmetry. If $\mathrm{d} \theta / \mathrm{d} \eta=0$, it follows from (1) and (2) that

$$
\frac{\mathrm{d} M}{d \eta}=0 \quad \text { and } \quad \theta=0 .
$$

The ray angles raise gradually from $\theta_{1}$ at the shockshock to $\theta_{\mathrm{w}}$ at the wall of the cone. We apply circular arcs which are perpendicular to the rays at the shock-shock and the wall, to approximate the shock shape (see Figure 4). Thus the configuration depends on the three parameters $\chi, \theta_{1}$ and $\theta_{\mathrm{w}}$; the length factor is not important because of selfsimilarity. 


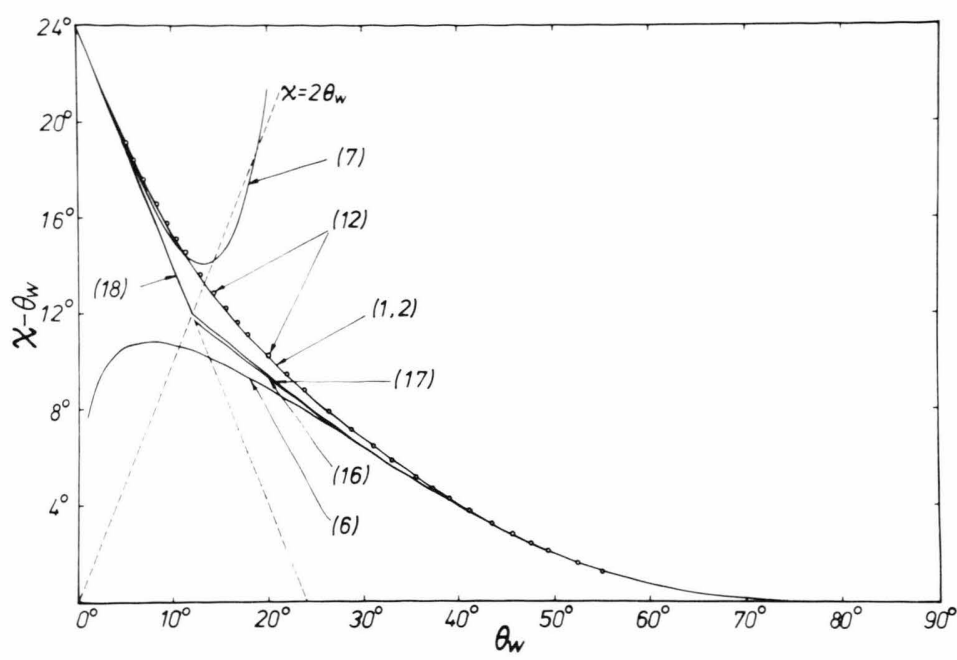

Fig. 3. Shock-shock angle $\chi$ vs. cone angle $\theta_{\mathrm{w}}$ for $M \gg 1$. The numbers represent those of the equations given in this paper.

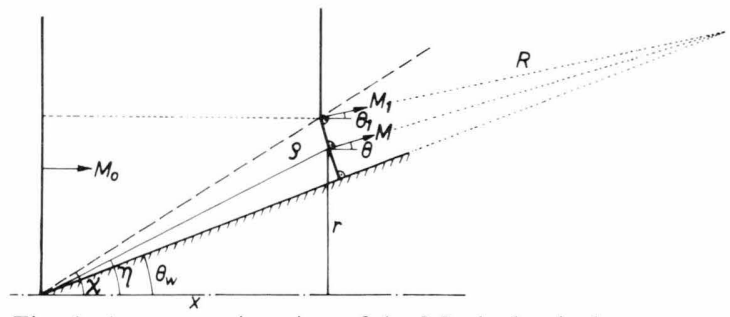

Fig. 4. Arc approximation of the Mach shock shape.

For the present approximation the following relations hold

$$
\begin{aligned}
& \frac{\sin (\eta-\theta)}{\sin \left(\eta-\theta_{\mathrm{w}}\right)}=\frac{\sin \left(\chi-\theta_{1}\right)}{\sin \left(\chi-\theta_{\mathrm{w}}\right)}:=K\left(\chi, \theta_{1}, \theta_{\mathrm{w}}\right) \\
& \left(\text { namely } \operatorname{tg} \eta=\frac{K \sin \theta_{\mathrm{w}}-\sin \theta}{K \cos \theta_{\mathrm{w}}-\cos \theta}\right), \\
& \frac{r}{R}=K \sin \theta_{\mathrm{w}}-\sin \theta, \\
& \frac{x}{R}=K \cos \theta_{\mathrm{w}}-\cos \theta, \\
& \frac{M}{M_{1}}=\frac{\sin \chi}{\sin \eta} \cdot \frac{\cos (\eta-\theta)}{\cos \left(\chi-\theta_{1}\right)} \cdot \frac{K \sin \theta_{\mathrm{w}}-\sin \theta}{K \sin \theta_{\mathrm{w}}-\sin \theta_{1}},
\end{aligned}
$$

where $R$ is the radius of the arc, $r$ the distance from the axis, and $x$ the coordinate in axial direction (see Figure 4). Equations (8) and (11) give the dependence of $\theta$ and $M$ with $\eta$, and (9) and (10) represent the shape of the Mach shock.
The problem is now reduced to determine the relation between the cone angle $\theta_{\mathrm{w}}$ and shock-shock parameters $\left(M_{1}, \chi, \theta_{1}\right)$.

From the definition of $A$ (see [1], [2])

$$
A \mathrm{~d} \beta=\mathrm{d} \Omega,
$$

where $\Omega$ is the area of the shock and $\beta$ is the one of Whitham's curved coordinates, tangential to the shock surface, i.e. $\beta=$ const. represent rays. Hence, integrating $\mathrm{d} \Omega / A$ from the ray at the wall of the cone to another one in the undisturbed and disturbed region, the same value is obtained:

$$
\frac{\pi r_{1}^{2}}{A_{0}}=\int_{\theta_{1}}^{\theta_{\mathrm{w}}} \frac{2 \pi r}{A} R \mathrm{~d} \theta,
$$

that is

$$
\frac{A_{1} r_{1}}{A_{0} R}=2 \int_{\theta_{1}}^{\theta_{\mathrm{w}}} \frac{A_{1}}{A} \frac{r}{r_{1}} \mathrm{~d} \theta .
$$

$A$ is a known function of $M$ (see (3)), and $M(\theta)$ and $r(\theta)$ are given by (8), (9), and (11). Hence this equation represents a relation between $M_{1}, \chi, \theta_{1}$ and $\theta_{\mathrm{w}}$. We may approximate the integral by the mean value of the integrand taken at the two boundaries:

$$
\frac{A_{1} r_{1}}{A_{0} R}=\left(1+\frac{A_{1}}{A_{\mathrm{w}}} \frac{r_{\mathrm{w}}}{r_{1}}\right)\left(\theta_{\mathrm{w}}-\theta_{1}\right),
$$

Substituting (4') and (9) into this relation yields

$$
\begin{aligned}
& \left(K \sin \theta_{\mathrm{w}}-\sin \theta_{1}\right) \frac{\sin \left(\chi-\theta_{1}\right)}{\sin \chi_{1}} \\
& =\left(1+\frac{A_{1}}{A_{\mathrm{w}}} \frac{(K-1) \sin \theta_{\mathrm{w}}}{K \sin \theta_{\mathrm{w}}-\sin \theta_{1}}\right)\left(\theta_{\mathrm{w}}-\theta_{1}\right),
\end{aligned}
$$


which represents the equation relating the cone angle $\theta_{\mathrm{w}}$ to the shock-shock parameters. $A$ is the known function of $M$, and from (11) follows

$$
\frac{M_{\mathrm{w}}}{M_{1}}=\frac{(K-1) \sin \chi}{\cos \left(\chi-\theta_{1}\right)\left(K \sin \theta_{\mathrm{w}}-\sin \theta_{1}\right)} .
$$

We have also plotted the calculated result for $M \gg 1$ as circles in Figure 3. It can be seen that it is in good agreement with the result from the numerical integration. Whitham [2] has given the result for $M_{1} / M_{0}=1.2(M \gg 1)\left(\theta_{1}=22.4^{\circ}, \chi=35.8^{\circ}\right): \theta_{\mathrm{w}}=$ $28.8^{\circ}$. Our result is $\theta_{\mathrm{w}}=28.7^{\circ}$. The distributions of $\theta$ and $M / M_{0}$ as functions of $\eta$ are obtained from (8) and (11), the shape of the shock is given by (9) and (10) or by the geometrical construction of the arc. Comparing with [2] (Figs. 6, 5 and 4) the agreement is very good (see Figs. 5, 6, and 7).

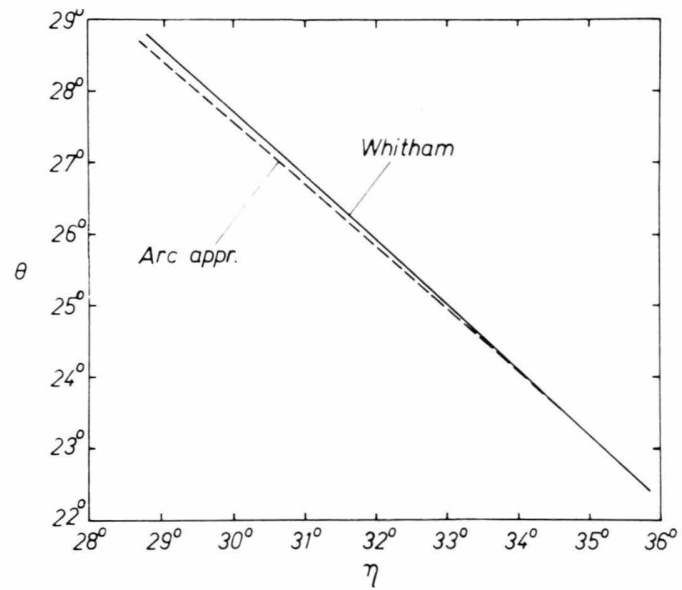

Fig. 5. Distribution of $\theta$ with $\eta$ for $M_{1} / M_{0}=1.2(M \gg 1)$.

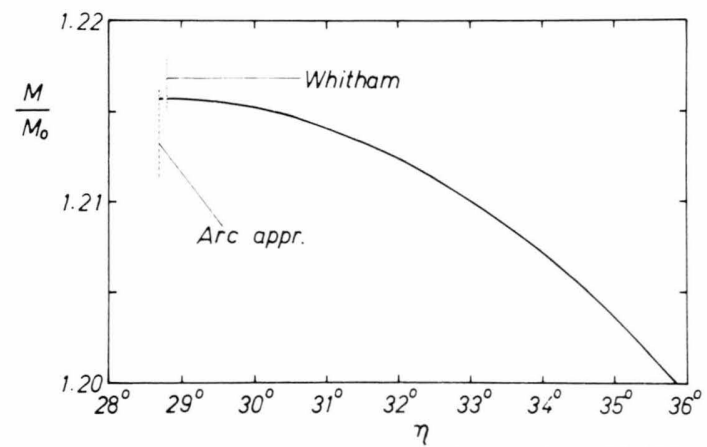

Fig. 6. Distribution of $M / M_{0}$ with $\eta$ for $M_{1} / M_{0}=1.2$ $(M \gg 1)$.

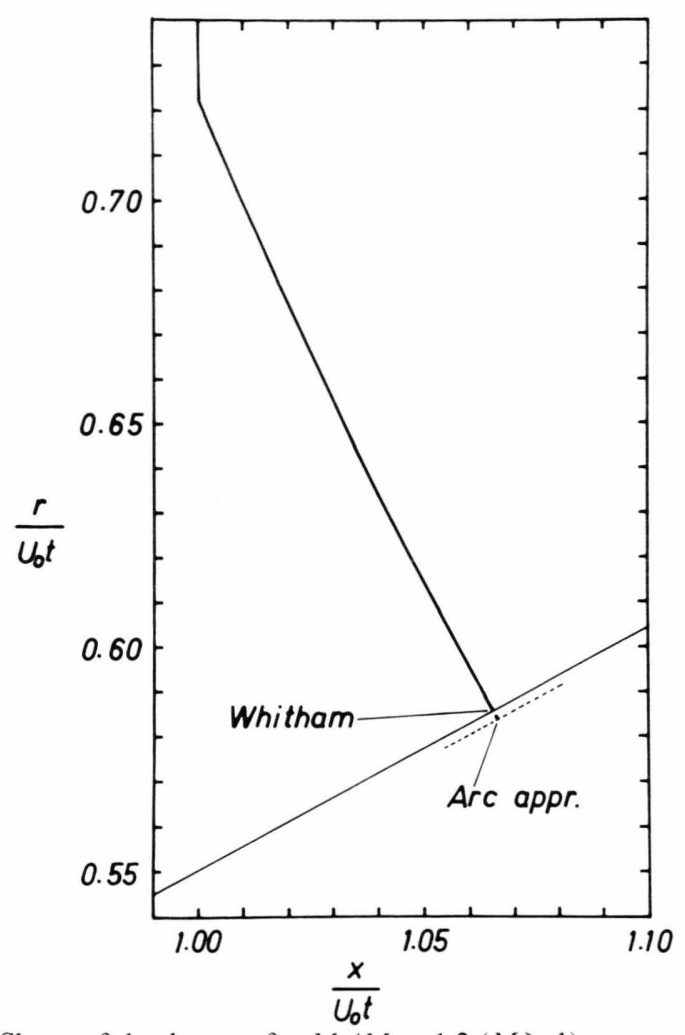

Fig. 7. Shape of shock wave for $M_{1} / M_{0}=1.2(M \gg 1)$.

\section{Precision of the arc Approximation}

At the wall of the cone $\eta=\theta=\theta_{\mathrm{w}}$. Thus it follows from (1) and (2) that

$$
\left.\begin{array}{l}
\mathrm{d} M / \mathrm{d} \eta=0 \\
\mathrm{~d} \theta / \mathrm{d} \eta=-1
\end{array}\right\} \quad \text { for } \quad \eta=\theta=\theta_{\mathrm{w}}
$$

For our arc approximation the first condition is satisfied automatically. If we obtain the equation which relates $\theta_{\mathrm{w}}$ and the shock-shock parameters from condition (15), it follows

$$
\sin \left(\chi-\theta_{1}\right)=2 \sin \left(\chi-\theta_{\mathrm{w}}\right) .
$$

The result calculated from (16) has also been plotted in Figure 3. Generally speaking, there is some error. It may be regarded that the arc approximation for the shock shape can have a precision up to the first order of differentiation (namely $\theta$ ). 


\section{Two-sided Approximation}

For $\theta_{1} \rightarrow 0$, i.e. $M_{1} \rightarrow M_{0}$, we denote $\chi=\chi_{0}$, which then depends only on $M_{0}$ and is identical with $m$ in Whitham's and Bryson's and Gross' papers. It represents the angle along which the shock perturbation propagates.

As $\theta_{1}=0, \theta_{\mathrm{w}}$ obtained from (16) is not equal to zero, hence (16) is only valid on the right-hand side of the median line in Figure 3.

If the approximation $\sin \alpha \approx \alpha$ is applied to (16), it becomes

$$
\chi+\theta_{1}=2 \theta_{\mathrm{w}}
$$

the difference between the two results from (16) and (17) is not large (see Figure 3).

For small cone angles, zero order approximation is applied, that means

$$
\chi=\chi_{0} \text { for any } \theta_{\mathrm{w}} .
$$

If one joins (17) and (18), the following two-sided approximation may be formulated

$$
\begin{aligned}
& \chi+\theta_{1}=2 \theta_{\mathrm{w}} \text { for } \theta_{\mathrm{w}} \geqq \chi_{0} / 2, \\
& \chi=\chi_{0} \quad \text { for } \quad \theta_{\mathrm{w}} \leqq \chi_{0} / 2 \text {. }
\end{aligned}
$$

$\theta_{\mathrm{w}}=\gamma_{0} / 2$ may be regarded as the boundary between small and large cone angels. The nearer $\theta_{\mathrm{w}}$ approaches to $\chi_{0} / 2$ the larger the error becomes.

The physical meaning of this two-sided approximation may be seen from Figure 8. In the region of large cone angles, $\theta_{\mathrm{w}}$ is related to $\left(\chi, \theta_{1}\right)$ at shockshock approximately by $\mathrm{d} \theta / \mathrm{d} \eta=-1$ (namely (17)); however, in the region of small cone angles, all points at the wall of the cone are related approximately to the same point $\theta=0, \chi=\chi_{0}$ at the shockshock (namely (18)).

\section{Conclusion}

For the axially symmetric diffraction problem of plane shocks by cones we may apply circle arcs to

1] G. B. Whitham, J. Fluid Mech. 2, 145 (1957).

[2] G. B. Whitham, J. Fluid Mech. 5, 369 (1959).

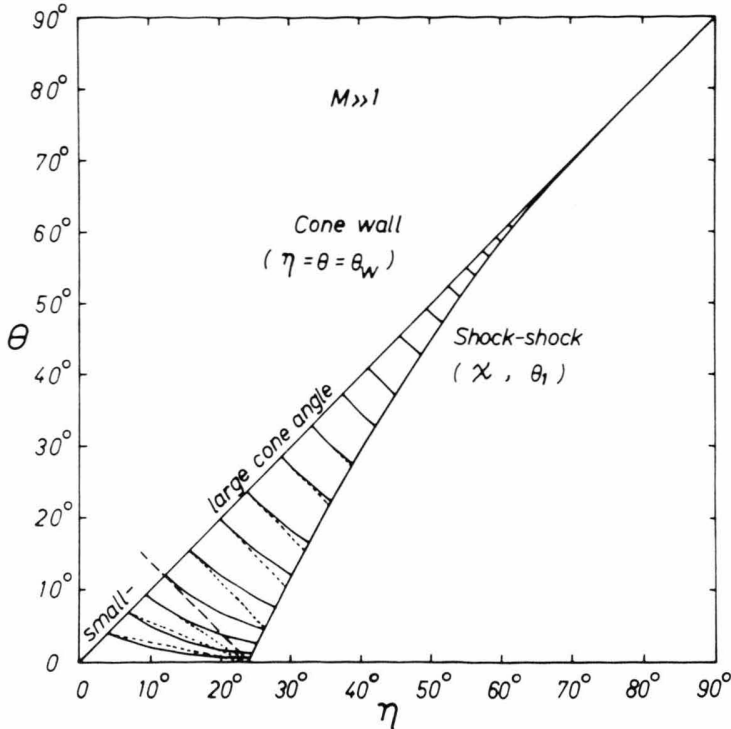

Fig. 8. Correspondence between cone angle and shockshock.

approximate the Mach shock shape. The distribution of ray angle $\theta$ and Mach number $M$ are obtained by (8) and (11), the shape of the Mach shock is determined by (9) and (10). Equation (12) with the addition of (13) and (3) relates the cone angle $\theta_{\mathrm{w}}$ with the shock-shock parameters. This approximation is very good.

Most simply, for a first assessment with nearly no calculation, the cone angle $\theta_{\mathrm{w}}$ may be related to the shock-shock parameters by (17) or (18). In the cases of small or large cone angles the approximation is good, but the nearer $\theta_{\mathrm{w}}$ approaches to $\chi_{0} / 2$ the larger the error becomes.

\section{Acknowledgement}

This work is part of a research project of the Sonderforschungsbereich 27 "Wave Focussing" at the Technical University Aachen sponsored by the Deutsche Forschungsgemeinschaft. This support is gratefully acknowledged.

[3] A. E. Bryson and R. W. F. Gross, J. Fluid Mech. 10, 1 (1961). 